\author{
LINEAR AND NON-LINEAR THEORY OF \\ GENERALIZED FUNCTIONS AND ITS APPLICATIONS \\ BANACH CENTER PUBLICATIONS, VOLUME 88 \\ INSTITUTE OF MATHEMATICS \\ POLISH ACADEMY OF SCIENCES \\ WARSZAWA 2010
}

\title{
HEAVISIDE'S THEORY OF SIGNAL TRANSMISSION ON SUBMARINE CABLES
}

\author{
HIKOSABURO KOMATSU \\ Graduate School of Mathematical Sciences, University of Tokyo \\ Komaba 3-8-1, Tokyo, 153-8914 Japan \\ E-mail: komatsu@ms.u-tokyo.ac.jp
}

\begin{abstract}
As written in L. Schwartz' book, Heaviside's theory of cables is an important source of the theory of generalized functions. The partial differential equations he discussed were the usual heat equation and the simplest hyperbolic equations of one space dimension, but he had to solve them as evolution equations in the unusual direction of the distance along which the electric signals propagate. Although he obtained explicit expressions of solutions, which were of great economical values, it has not yet been clarified completely how he derived and proved them. The author gives easy proofs of Heaviside's results based only on the classical theory of Laplace transforms and their reciprocal, the Bromwich integrals. At the end it is indicated that an abstract version of the Fatou theorem on bounded harmonic functions on a half space implies the uniqueness of solutions for the Thomson cables.
\end{abstract}

1. Fourier's $\delta$-function and operational calculus. Schwartz' book "Théorie des distributions" 25] starts with citations of Heaviside's paper [10] on operational calculus and Dirac's [5] on quantum mechanics. These are supposed to be the first places where distributions were actually employed.

It is little known, however, that Joseph Fourier (1768-1830) introduced the $\delta$-function much earlier as an integral kernel, and formulated his integral formula as

$$
\delta(x-\alpha)=\frac{1}{\pi} \int_{0}^{\infty} \cos (q(x-\alpha)) d q .
$$

His book "Théorie analytique de la chaleur" 7 claims this repeatedly. In particular, the statement on p. 449 is incontestable. He also gives many proofs of (1) and, in particular, the last one given on pp. 546-551 is essentially the same as that of Dirichlet, Riemann

2000 Mathematics Subject Classification: Primary 44A45; Secondary 35C15.

Key words and phrases: Heaviside, square roots of differential operators.

The paper is in final form and no version of it will be published elsewhere. 
and Jordan and is sufficient for developing his "fonctions quelconques" which are actually piecewise real analytic functions.

Moreover, Fourier has developed on pp. 511-546 an operational calculus in order to solve not only the initial value problem of the heat equation but also many other linear partial differential equations.

He starts with the expansion of a general solution into a formal power series, and then writes the result in an operational expression. For example, consider the initial value problem of the heat equation:

$$
\left\{\begin{array}{l}
\frac{\partial v}{\partial t}=\frac{\partial^{2} v}{\partial x^{2}}, \quad t>0, x \in \mathbb{R} \\
v(0, x)=\phi(x), \quad x \in \mathbb{R}
\end{array}\right.
$$

Then, we have

$$
v(t, x)=\phi(x)+t \phi^{(2)}(x)+\frac{t^{2}}{2 !} \phi^{(4)}(x)+\frac{t^{3}}{3 !} \phi^{(6)}(x)+\cdots,
$$

which may be written $e^{t D^{2}} \phi(x)$ with $D=\partial / \partial x$.

Applying $e^{t D^{2}}$ to $\cos (q(x-\alpha))$, we get

$$
e^{t D^{2}} \cos (q(x-\alpha))=e^{-t q^{2}} \cos (q(x-\alpha)) .
$$

Fourier obtains the heat kernel as its integral with respect to $q$ :

$$
\frac{1}{2 \pi} \int_{-\infty}^{\infty} e^{-t q^{2}} \cos (q(x-\alpha)) d q=\frac{1}{2 \sqrt{\pi t}} e^{-\frac{(x-\alpha)^{2}}{4 t}} .
$$

His proof given on p. 477 makes use of a shift of the path of integration in

$$
\sqrt{\pi}=\int_{-\infty}^{\infty} e^{-q^{2}} d q
$$

In his book [7] Fourier attributes the integral formula

$$
v(t, x)=\frac{1}{2 \sqrt{\pi t}} \int_{-\infty}^{\infty} e^{-\frac{(x-\alpha)^{2}}{4 t}} \phi(\alpha) d \alpha=\frac{1}{\sqrt{\pi}} \int_{-\infty}^{\infty} e^{-q^{2}} \phi(x+2 q \sqrt{t}) d q
$$

of solutions to (2) to Laplace in the latter form but he never forgets to mention that Laplace's paper appeared later than the year 1807 in which he submitted the first part of his book to the Academy (and its committee with Laplace as a member rejected to publish it).

Along with the usual initial value problem (2), Fourier considers also the initial value problem

$$
\left\{\begin{array}{l}
\frac{\partial^{2} v}{\partial x^{2}}=\frac{\partial v}{\partial t}, \quad t, x \in \mathbb{R}, \\
v(t, 0)=\phi(t), \quad t \in \mathbb{R}, \\
\frac{\partial}{\partial x} v(t, 0)=\psi(t), \quad t \in \mathbb{R},
\end{array}\right.
$$


in the direction of space. In this case, we have

$$
\begin{aligned}
v(t, x)= & \phi(t)+\frac{x^{2}}{2 !} \phi^{\prime}(t)+\frac{x^{4}}{4 !} \phi^{(2)}(t)+\frac{x^{6}}{6 !} \phi^{(3)}(t)+\cdots \\
& +x \psi(t)+\frac{x^{3}}{3 !} \psi^{\prime}(t)+\frac{x^{5}}{5 !} \psi^{(2)}(t)+\frac{x^{7}}{7 !} \psi^{(3)}(t)+\cdots \\
= & \cos (x \sqrt{-\partial / \partial t}) \phi(t)+\frac{\sin (x \sqrt{-\partial / \partial t})}{\sqrt{-\partial / \partial t}} \psi(t) .
\end{aligned}
$$

Power series (4) converges if the functions $\phi$ and $\psi$ are in the Gevrey class of index (2), i.e., if their $m$-th derivatives are bounded by $C_{\epsilon} \epsilon^{m} m !^{2}$ for any $\epsilon>0$ with a constant $C_{\epsilon}$ (see [14]).

On p. 544 Fourier gives an integral formula of the fundamental solution. It converges as an ultradistribution of class (2) but never as a function in any sense.

In the same way, the solution of the initial value problem of the wave equation in two dimensions is expressed as

$$
u(t, x, y)=\cos (t \sqrt{-\Delta}) \phi(x, y)+\frac{\sin (t \sqrt{-\Delta})}{\sqrt{-\Delta}} \psi(x, y) .
$$

Fourier almost stops here but it is not difficult hence to obtain the explicit integral formula of solutions due to Poisson and Herglotz.

2. Thomson's theory of cables. A similar problem appears in the theory of signal transmission on submarine cables. Telegraphy was invented in 1837 by C. Wheatstone in England and by S. F. B. Morse in the United States independently. Long distance telecommunication systems were of vital importance for both countries to maintain their huge territories. Already in 1850 they started to construct submarine cables for telegraphy but first cables didn't show their expected performance. Signals were so blurred that they became illegible at the receivers.

In 1855 William Thomson (1824-1907) published the first paper [28] analyzing the signal transmission on long cables. The paper is composed of three extracts from correspondence he exchanged with G. G. Stokes.

The electric property of cables or parallel wires may be approximated by the following circuit. We put an electro-motive force $\phi(t)$ at one end of a cable and observe the electric current $j(t)$ at the other end.

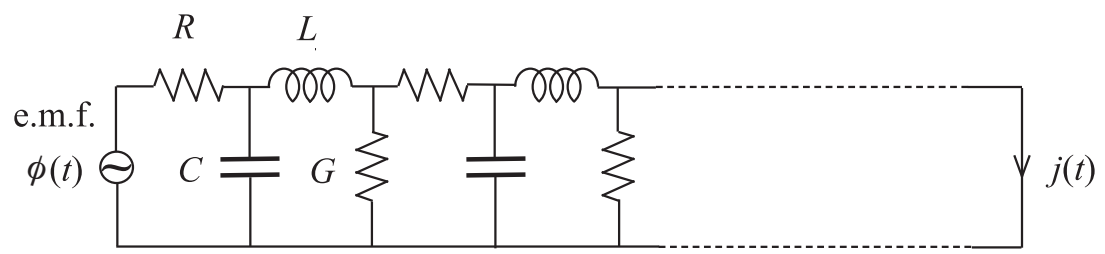

Thomson took into consideration only the electrostatic capacitance $C$ between the wire and the sheath and the electric resistance $R$ of the wire, and ignored the selfinductance $L$ and the leakage conductance $G$. Let $C$ and $R$ be the capacitance and the 
resistance per unit length, respectively. Then, the voltage $v(t, x)$ and the current $j(t, x)$ of the wire obey the equations

$$
-\frac{\partial v}{\partial x}=R j, \quad-\frac{\partial j}{\partial x}=C \frac{\partial v}{\partial t} .
$$

For the sake of simplicity we consider an infinitely long cable $x \geq 0$. Let the cable be quiet for $t<0$. Then, we put an electro-motive force $\phi(t)$ at $x=0$ and observe the electric current $j(t, x)$ at $x>0$. Eliminating $j$, we have the following problem:

$$
\left\{\begin{array}{l}
\frac{\partial^{2} v(t, x)}{\partial x^{2}}=C R \frac{\partial v(t, x)}{\partial t}, \quad x \geq 0 \\
v(t, x)=0, \quad t<0, x \geq 0 \\
v(t, 0)=\phi(t), \quad t \geq 0
\end{array}\right.
$$

The solution Thomson and Stokes gave is symbolically

$$
v(t, x)=e^{-\sqrt{C R} x \sqrt{d / d t}} \phi(t)
$$

and more concretely

$$
=\frac{1}{2 \sqrt{\pi}} \int_{0}^{t} \frac{\sqrt{C R} x}{(t-s)^{3 / 2}} e^{-\frac{C R x^{2}}{4(t-s)}} \phi(s) d s .
$$

If, in particular, $\phi(t)$ is the Heaviside function

$$
\theta(t)= \begin{cases}1, & t \geq 0 \\ 0, & t<0\end{cases}
$$

then we have

$$
v(t, x)=1-\operatorname{erf}\left(\frac{x}{2} \sqrt{\frac{C R}{t}}\right)=\frac{2}{\sqrt{\pi}} \int_{\frac{x}{2} \sqrt{\frac{C R}{t}}}^{\infty} e^{-u^{2}} d u .
$$

Except for the coefficient $C R$ the differential equation in Thomson's problem (5) is the same as Fourier's (3), but the numbers of the initial values are one for (5) and two for (3). The solutions expressed by (7) represent waves going in the positive direction in $x$, and there are also waves going in the opposite direction. We cannot exclude the latter type of solutions without the quietness condition in the middle of (5). We remark, however, that Fourier's solutions (4) satisfy the quietness condition if $\phi(t)=\psi(t)=0$ for $t<0$. This shows that physical phenomena cannot be described by differential equations only. For Thomson's problem (5) it is intuitively clear that we have only waves going in the positive direction, so that only one initial condition is allowed. This intuitive fact is not necessarily explained clearly in a mathematical or physical language. Heaviside relied on the causality as shown later. We may also appeal to the dissipation of energy or the like (see Section 6).

To obtain (8), Thomson made use of the formula

$$
\int_{0}^{\infty} e^{-z \sqrt{n}} \cos (2 n t-z \sqrt{n}) d n= \begin{cases}\frac{\sqrt{\pi} z}{4 \sqrt{t^{3}}} e^{-\frac{z^{2}}{4 t}}, & t>0 \\ 0, & t \leq 0 .\end{cases}
$$


In order to make its meaning clearer, we change the variable into $2 n=\omega$. Then, we have

$$
\frac{1}{2} \int_{0}^{\infty} e^{-\frac{z \sqrt{\omega}}{\sqrt{2}}} \cos \left(\omega t-\frac{z \sqrt{\omega}}{\sqrt{2}}\right) d \omega=\frac{1}{4} \int_{-\infty}^{\infty} e^{-z \sqrt{i \omega}} e^{i \omega t} d \omega,
$$

where

$$
\sqrt{i \omega}= \begin{cases}\frac{1+i}{\sqrt{2}} \sqrt{\omega}, & \omega \geq 0 \\ \frac{1-i}{\sqrt{2}} \sqrt{-\omega}, & \omega<0 .\end{cases}
$$

Thus formula $\sqrt{9}$ is equivalent to

$$
\frac{1}{2 \pi} \int_{-\infty}^{\infty} e^{-z \sqrt{i \omega}} e^{i \omega t} d \omega= \begin{cases}\frac{z}{\sqrt{4 \pi t^{3}}} e^{-\frac{z^{2}}{4 t}}, & t>0 \\ 0, & t \leq 0\end{cases}
$$

for $z>0$.

This may be the most valuable mathematical result in the nineteenth century in the sense that the discoverer got the most wealth.

Thomson did not give the details how he computed it but we can find in the paper [28] Stokes' complicated proof based on the Fourier sine transformation. Expression (7) of a general solution is also due to Stokes.

In 1866 a transatlantic cable was successfully constructed after Thomson's design and he was knighted for his contributions. In 1892 he became Lord Kelvin, Baron of Largs.

3. Heaviside's operational calculus. Oliver Heaviside (1850-1925) is an Englishman born in a poor family and had no formal education of college level. Since his mother was a sister of C. Wheatstone's wife, he was able to get a position of telegraphist but resigned at the age of 24 and spent the rest of his life in writing mathematical and physical papers for a weekly commercial newspaper "The Electrician". His theory of operational calculus [9, 10, 11] was invented first to make the Fourier-Thomson theory more accessible to engineers.

After we have an operational representation like (6), we have to carry out difficult integration in order to obtain the solution $v(t, x)$ or the integral kernel $(\mathrm{T})$. Heaviside employed instead the fractional power series expansions in the operator $p=d / d t$ and computed the integrals as series of distributions with the use of the following correspondence of operators and distributions under the causality condition:

$$
p^{\alpha} \longleftrightarrow p^{\alpha} \delta(t)=\left\{\begin{array}{cc}
\frac{t_{+}^{-\alpha-1}}{\Gamma(-\alpha)}, & \alpha \neq 0,1,2, \ldots, \\
\delta^{(\alpha)}(t), & \alpha=0,1,2, \ldots,
\end{array}\right.
$$

where $t_{+}^{-\alpha-1}$ denotes Hadamard's finite part of the function $t^{-\alpha-1}$ for $t>0$ and 0 for $t<0$ [20].

For example, Thomson's problem (5) is solved as follows:

$$
\begin{aligned}
v(t, x) & =e^{-\sqrt{R C} x \sqrt{p}} \phi(t)=\left(1-q x+\frac{(q x)^{2}}{2 !}-\frac{(q x)^{3}}{3 !}+\cdots\right) \phi(t) \\
& =(\cosh q x-\sinh q x) \phi(t)
\end{aligned}
$$


where

$$
q=\sqrt{C R} \sqrt{p}=\sqrt{C R} \sqrt{d / d t} .
$$

To compute the integral kernel $(\mathrm{T})$, let $\phi(t)$ be the delta function $\delta(t)$. Then, $\cosh q x$ being a differential operator (of infinite order), we have

$$
(\cosh q x) \delta(t)=0, \quad t \neq 0 .
$$

On the other hand, we have

$$
\begin{aligned}
\left(\frac{d}{d t}\right)^{m+\frac{1}{2}} \delta(t) & =\left(\frac{d}{d t}\right)^{m+1}\left(\frac{d}{d t}\right)^{-\frac{1}{2}} \delta(t) \\
& =\frac{1}{\sqrt{\pi}}\left(\frac{d}{d t}\right)^{m+1} \frac{1}{\sqrt{t_{+}}}=\frac{(-1)^{m+1}}{2 \sqrt{\pi}} \frac{(2 m+1) !}{m ! 4^{m} t_{+}^{m+\frac{3}{2}}}
\end{aligned}
$$

Hence it follows that $-(\sinh q x) \delta(t)=0$ for $t<0$ and that

$$
\begin{aligned}
-(\sinh q x) \delta(t) & =-\sqrt{C R} x \sum_{m=0}^{\infty} \frac{\left(C R x^{2}\right)^{m}}{(2 m+1) !}\left(\frac{d}{d t}\right)^{m+\frac{1}{2}} \delta(t) \\
& =\frac{\sqrt{C R} x}{2 \sqrt{\pi t^{3}}} \sum_{m=0}^{\infty} \frac{1}{m !}\left(-\frac{C R x^{2}}{4 t}\right)^{m}=\frac{\sqrt{C R} x}{2 \sqrt{\pi t^{3}}} e^{-\frac{C R x^{2}}{4 t}} \quad \text { for } t>0 .
\end{aligned}
$$

This is his method which Schwartz commented as 'audacieux' in [25]. It was more so at the end of the nineteenth century. Heaviside's paper [10] originally consisted of three parts. After two parts were published, the third one was rejected as nonsense and was never published. He was almost ignored in his life, but soon after his death people began to evaluate him. Whittaker [29] wrote in 1928:

Looking back on the controversy after thirty years, we should now place the Operational Calculus with Poincaré's discovery of automorphic functions and Ricci's discovery of the Tensor Calculus as the three most important mathematical advances of the last quarter of the nineteenth century.

Many books have been published since then on its applications, extensions and justifications, e.g., van der Pol-Bremmer [24], Jeffreys [12] and Mikusiński [23. However, their treatments are not necessarily so straightforward as Heaviside.

Today we can righteously interpret most of Heaviside's results with the help of the theory of hyperfunctions and ultradistributions [15]-[20]. In the above case, for example, $\cosh q x$ and $\sinh q x$ are an ultradifferential operator of class (2) and a pseudodifferential operator of class (2), respectively, so that the series converge as ultradistributions of class (2) and coincide with the above sums on $\{t: t \neq 0\}$. On the other hand, since $e^{-z \sqrt{i \tau}}$ is integrable as a function in $\tau$, integral ( $\mathrm{T}$ ) is a continuous function in $t$. Thus, we have the identity $(\mathrm{T})$.

Heaviside made use of his operational calculus also to establish the theory of cables for telephone. Telephony had been invented by A. G. Bell in 1876. Heaviside knew from his experience that the leakage and the self-induction of cables improved their performance at high frequencies in spite of all our intuition. He was also one of the very few who understood the electromagnetic theory of J. C. Maxwell (1831-1879) at that 
time and was able to compute the self-inductance of cables, which was by no means negligible.

Let $L$ and $G$ be the inductance and the leakage conductance per unit length. Then, he showed in 1881 that the voltage $v$ and the current $j$ obey

$$
\left\{\begin{array}{l}
-\frac{\partial v}{\partial x}=R j+L \frac{\partial j}{\partial t}, \\
-\frac{\partial j}{\partial x}=G v+C \frac{\partial v}{\partial t}
\end{array}\right.
$$

and hence the differential equation for $v$ is

$$
\frac{\partial^{2} v}{\partial x^{2}}=\left(R+L \frac{\partial}{\partial t}\right)\left(G+C \frac{\partial}{\partial t}\right) v .
$$

This is usually called the telegraphist equation but the Heaviside equation would be a more appropriate name.

Thus the problem of signal transmission on cables is reduced to that of finding solution of (10) under conditions

$$
\left\{\begin{array}{l}
v(t, x)=0, \quad t<0, \quad x \geq 0, \\
v(t, 0)=\phi(t), \quad t \geq 0
\end{array}\right.
$$

The following are the most important results Heaviside obtained in the case $L C \neq$ 0 . Both were announced in the year 1887, that is, the memorable 50th anniversary of telegraphy.

Distortionless Cables: If condition

$$
C R=L G
$$

is satisfied, then equation 10 is reduced to

$$
\frac{\partial^{2} v}{\partial x^{2}}=L C\left(\frac{\partial}{\partial t}+\frac{R}{L}\right)^{2} v
$$

and its solution is

$$
v(x, t)=e^{-\sqrt{R G} x} \phi(t-\sqrt{L C} x) .
$$

This shows that the signal attenuates exponentially according to the distance but it propagates without distortion.

General Cables: Assume

$$
C R \neq L G
$$

Then, equation 10 is written

$$
\frac{\partial^{2} v}{\partial x^{2}}=L C\left\{\left(\frac{\partial}{\partial t}+\rho\right)^{2}-\sigma^{2}\right\} v
$$

with

$$
\rho=\frac{C R+L G}{2 L C}, \quad \sigma=\frac{|C R-L G|}{2 L C} .
$$


Its solution is given by

$$
\begin{aligned}
v(x, t) & =e^{-\rho \sqrt{L C} x} \phi(t-\sqrt{L C} x) \\
& +\int_{0}^{t-\sqrt{L C} x} \frac{e^{-\rho(t-s)} \sigma \sqrt{L C} x}{\sqrt{(t-s)^{2}-L C x^{2}}} I_{1}\left(\sigma \sqrt{(t-s)^{2}-L C x^{2}}\right) \phi(s) d s,
\end{aligned}
$$

where $I_{1}$ is the modified Bessel function of order 1 .

He should have thought that then he surpassed Sir William. The response was, however, quite opposite. He was declined to contribute his papers to "The Electrician". He lost his steady income. Fortunately, Heinrich Hertz (1857-1894) published in 1888 papers on his famous experiments, and Heaviside survived.

He must have been very much proud of his discovery of the distortionless condition. Since the capacitance $C$ is too large because of high electrostatic inductivity of insulators, he proposed plans to increase the inductance $L$. Finally, loaded cables, which have coils at every fixed distance, have been widely utilized. But the American patent went to Pupin in 1900. Pupin became a millionaire but Heaviside had nothing and died of poverty. In 1932 Shigeyoshi Matsumae invented the method of sending multi-channeled signals carried by waves of high frequency on unloaded cables. The income he got from his patent was large enough to create an engineering college, which is now Tokai University.

Heaviside's strategy is the same as above. Equation 13 is written

$$
\frac{\partial^{2} v}{\partial x^{2}}=L C\left\{(p+\rho)^{2}-\sigma^{2}\right\} v=q^{2} v,
$$

where

$$
q=\sqrt{L C} \sqrt{(p+\rho)^{2}-\sigma^{2}}
$$

Hence its solution should be

$$
v=e^{q x} A+e^{-q x} B,
$$

with constants $A$ and $B$ in the sense that they are independent of $x$. The attenuation rate $\rho$ may be eliminated by the general rule

$$
F(p+\rho) v=e^{-\rho t} F(p)\left(e^{\rho t} v\right) .
$$

In case $\sigma=\mathbf{0}$, the general solution is, therefore,

$$
\begin{aligned}
v & =e^{-\rho t} e^{\sqrt{L C} x p}\left(e^{\rho t} A\right)+e^{-\rho t} e^{-\sqrt{L C} x p}\left(e^{\rho t} B\right) \\
& =e^{\rho \sqrt{L C} x} A(t+\sqrt{L C} x)+e^{-\rho \sqrt{L C} x} B(t-\sqrt{L C} x),
\end{aligned}
$$

where $A$ and $B$ are arbitrary functions in $t$. The first term represents a wave going to the left, which must vanish by the quietness condition for $t<0$. Thus, only the second term remains and gives the solution 12 .

In the above we have also employed the translation rule

$$
e^{z p} f(t)=f(t+z) \text {, for real } z \text {. }
$$

This is the Taylor expansion theorem but we know that it converges only for very special functions $f(t)$. That means that his method of power series expansions in $p$ does not work in the literal sense. 
In the general case, he also claims that

$$
v(t, x)=e^{-\sqrt{L C} x \sqrt{(p-\rho)^{2}-\sigma^{2}}} \phi(t)
$$

is the solution to problem (10) and (11) and obtains the true solution (14). By the Duhamel principle, we have only to know the response to the $\delta$-function $\delta(t)$ or the Heaviside function $\theta(t)$ and he mainly works for his unit function $\theta(t)$.

He was not allowed to publish his first proof (or heuristics). Judging from his announcement, it seems that he decomposed the exponential in 16 into the sum of sinh and cosh and computed their actions as in the case of the Thomson cables. Chapter VII of [11] is devoted to this problem. Considering the initial values of the current, he shows that if we take

$$
e^{-\rho t} I_{0}\left(\sigma t_{+}\right)=\frac{1}{\sqrt{(p+\rho)^{2}-\sigma^{2}}} \delta(t)
$$

as the initial value of $v$, the computation goes well.

J. R. Carson, who published the first understandable proof [4], writes "Heaviside's own attack on this problem from the operational equation, while distinguished by extraordinary ingenuity and almost uncanny intuition, can hardly be regarded as entirely satisfactory".

4. Laplace's integrals and Bromwich's. The first foundation for operational calculus was established by Bromwich [3], Carson [4] and Lévy [22] by the following correspondence between operators $F(p)$ and functions $f(t)=F(p) \delta(t)$ :

$$
\begin{gathered}
F(p)=\mathcal{L} f(p)=\int_{0}^{\infty} e^{-p t} f(t) d t \\
F(p) \delta(t)=\mathcal{L}^{-1} F(t)=\frac{1}{2 \pi i} \int_{c-\infty}^{c+\infty} e^{p t} F(p) d p
\end{gathered}
$$

the integral being evaluated along any line parallel to the imaginary axis with a sufficiently large $c$ (cf. [12] or [24]).

They are reciprocal. If $F(p)$ is the Laplace transform 17$)$ of $f(t)$, then the Bromwich integral 18 is equal to the original function $f(t)$, and vice versa. Of course we need conditions to guarantee the convergence of integrals.

The correspondence has been extended to the case where $f(t)$ are distributions by Schwartz [26] and Sebastião e Silva [27] and to hyperfunctions by Komatsu [15]-[20].

On the other hand, Mikusiński [23] established a completely different foundation of operational calculus by defining the operators to be elements in the quotient field of the convolution algebra of continuous functions. They include the ultradistributions [16]. Thus, a linear (ultra)differential equation has always a unique solution as an operator by "division". But its identification with a function or an ultradistribution is difficult. This is especially the case when the symbol of an operator is expressed as the exponential of an operator of the first order as in 15 or (16). Heaviside's naive method of fractional power series expansions does not work because the corresponding series of distributions do not converge in any localizable topology. Nevertheless, Mikusiński [23] overcame the 
difficulty of identifying solution (16) of the Heaviside cables with expression (14) by a four pages long calculation.

5. Our proofs. We give here rigorous but easy proofs to the above results of Thomson and Heaviside relying only on classical Laplace's and Bromwich's integrals.

The signal transmission on the Thomson cables has been reduced to the formula

$$
\frac{1}{2 \pi i} \int_{c-i \infty}^{c+i \infty} e^{\tau t} e^{-z \sqrt{\tau}} d \tau= \begin{cases}\frac{z}{\sqrt{4 \pi t^{3}}} e^{-\frac{z^{2}}{4 t}}, & t>0 \\ 0, & t \leq 0\end{cases}
$$

for $z>0$. It is easy to see that the value is 0 for $t<0$ by taking the limit as $c \rightarrow \infty$. The value at $t=0$ is also 0 by Lebesgue's convergence theorem. However, for $t>0$, proofs in the literature are rather complicated.

The following is an elementary proof by a senior student Toshiyuki Suzuki of Tokyo University of Sciences, who submitted it as a paper for the assignments of my course on operational calculus for this spring.

We change the independent variable $\tau$ in (T) into $\sqrt{\tau}=w$ and let $\Gamma$ be the image of the line of integration under this transformation. Then the left hand side of $(\mathrm{T})$ is equal to

$$
\begin{aligned}
& \frac{1}{2 \pi i} \int_{\Gamma} e^{w^{2} t} e^{-z w} 2 w d w=\frac{-1}{\pi i} \int_{\Gamma} \frac{\partial}{\partial z}\left(e^{w^{2} t-z w}\right) d w \\
& =\frac{\partial}{\partial z} \frac{-1}{\pi i} \int_{-i \infty}^{i \infty} e^{w^{2} t-z w} d w=-2 \frac{\partial}{\partial z} \frac{1}{2 \sqrt{\pi t}} e^{-\frac{z^{2}}{4 t}}=\frac{z}{\sqrt{4 \pi t^{3}}} e^{-\frac{z^{2}}{4 t}}
\end{aligned}
$$

To discuss the Heaviside Cables, we first compute the fundamental solution of the Klein-Gordon equation:

$$
\frac{\partial^{2}}{\partial t^{2}} u(t, x)=\left(\frac{\partial^{2}}{\partial x^{2}}+\sigma^{2}\right) u(t, x), \text { for a } \sigma>0,
$$

with support in the positive light cone $\left\{(t, x) \in \mathbb{R}^{2}: t \geq|x|\right\}$.

We write

$$
A=\frac{d^{2}}{d x^{2}}+\sigma^{2}
$$

and regard it as a closed linear operator in a suitable Banach space $E$ of functions on $\mathbb{R}$ on which the integrable functions in $L^{1}(\mathbb{R})$ act continuously by convolutions, e.g. $L^{p}(\mathbb{R})$ for $1 \leq p \leq \infty$. Then, the initial value problem

$$
\left\{\begin{array}{l}
\left(\frac{d^{2}}{d t^{2}}-A\right) u(t)=f(t), \\
u(0)=g_{0}, \quad u^{\prime}(0)=g_{1},
\end{array}\right.
$$

is well-posed if the inverse Laplace transform

$$
\mathcal{L}_{t}^{-1}\left(\tau^{2}-A\right)^{-1}=(\sin t \sqrt{-A}) / \sqrt{-A} \delta(t)
$$

exists and its kernel becomes a fundamental solution in $t$ and $x$ [18. 
In our case, the resolvent $\left(\tau^{2}-A\right)^{-1}$ is the convolution operator with the integrable kernel

$$
\frac{1}{2 \sqrt{\tau^{2}-\sigma^{2}}} e^{-\sqrt{\tau^{2}-\sigma^{2}}|x|} .
$$

Thus the fundamental solution $K(t, x)$ is computed as the integral

$$
K(t, x)=\frac{1}{2 \pi i} \int_{c-i \infty}^{c+i \infty} e^{\tau t} \frac{1}{2 \sqrt{\tau^{2}-\sigma^{2}}} e^{-\sqrt{\tau^{2}-\sigma^{2}}|x|} d \tau .
$$

By the usual argument as above we have $K(t, x)=0$ for $t \leq 0$. If $t>0$, we can deform the path of integration into a sufficiently large circle containing a slit connecting $\pm \sigma$ outside which the integrand is holomorphic. Hence it follows that $K(t, x)$ is analytic for $t>0$.

If $x=0$, it is well known ([24] Chap. X.4, [12] Chap. 21.03 or [20] $§ 5)$ that

$$
K(t, 0)=\mathcal{L}_{t}^{-1}\left(\frac{1}{2 \sqrt{\tau^{2}-\sigma^{2}}}\right)=\frac{1}{2} I_{0}\left(\sigma t_{+}\right),
$$

where

$$
I_{0}\left(t_{+}\right)= \begin{cases}I_{0}(t), & t>0 \\ 0, & t \leq 0\end{cases}
$$

and

$$
I_{0}(t)=1+\frac{t^{2}}{2^{2}}+\frac{t^{4}}{2^{2} 4^{2}}+\frac{t^{6}}{2^{2} 4^{2} 6^{2}}+\cdots
$$

is the modified Bessel function of order 0 .

The Klein-Gordon equation is Lorentz invariant, and so is its fundamental solution. To prove this it is enough to show that it is invariant under the infinitesimal Lorentz transformation. In fact, it follows from the Heaviside rules that

$$
\left(x \frac{\partial}{\partial t}+t \frac{\partial}{\partial x}\right) K(t, x)=\mathcal{L}_{t}^{-1}\left\{\left(x \tau-\frac{\partial}{\partial \tau} \frac{\partial}{\partial x}\right) \frac{1}{2 \sqrt{\tau^{2}-\sigma^{2}}} e^{-\sqrt{\tau^{2}-\sigma^{2}}|x|}\right\}=0 .
$$

Thus we have proved that the fundamental solution $K(t, x)$ is equal to

$$
\mathcal{L}_{t}^{-1}\left(\frac{1}{2 \sqrt{\tau^{2}-\sigma^{2}}} e^{-\sqrt{\tau^{2}-\sigma^{2}}|x|}\right)=\frac{1}{2} I_{0}\left(\sigma \sqrt{t^{2}-x^{2}}\right) \theta(t-|x|) .
$$

Since

$$
e^{-\sqrt{\tau^{2}-\sigma^{2}} x}=-2 \frac{\partial}{\partial x} \frac{1}{2 \sqrt{\tau^{2}-\sigma^{2}}} e^{-\sqrt{\tau^{2}-\sigma^{2}} x}
$$

we have

$$
\begin{aligned}
\mathcal{L}_{t}^{-1} e^{-\sqrt{\tau^{2}-\sigma^{2}} x} & =-2 \frac{\partial}{\partial x} \mathcal{L}_{t}^{-1} \frac{1}{2 \sqrt{\tau^{2}-\sigma^{2}}} e^{-\sqrt{\tau^{2}-\sigma^{2}} x} \\
& =-2 \frac{\partial}{\partial x} \frac{1}{2} I_{0}\left(\sigma \sqrt{t^{2}-x^{2}}\right) \theta(t-|x|) \\
& =\delta(t-x)+\frac{\sigma x}{\sqrt{t^{2}-x^{2}}} I_{1}\left(\sigma \sqrt{t^{2}-x^{2}}\right) \theta(t-|x|), \quad x>0,
\end{aligned}
$$

where

$$
I_{1}(t)=I_{0}^{\prime}(t)=\frac{t}{2}+\frac{t^{3}}{2^{2} 4}+\frac{t^{5}}{2^{2} 4^{2} 6}+\frac{t^{7}}{2^{2} 4^{2} 6^{2} 8}+\cdots
$$


Substituting $\sqrt{L C} x$ for $x$ in 21 , and multiplying it by the attenuation factor $e^{-\rho t}$, we obtain the kernel for the representation (14) of solutions.

Finally the Poisson integral

$$
P(t) \phi(x)=e^{-t \sqrt{-\Delta}} \phi(x), \quad t>0,
$$

is another important integral involving the exponential of the square root of a differential operator. Its integral kernel is given by the absolutely convergent integral

$$
P_{n}(t, x)=\frac{1}{(2 \pi)^{n}} \int_{\mathbb{R}^{n}} e^{-t|\xi|} e^{i x \xi} d \xi=\frac{2}{\omega_{n+1}} \frac{t}{\left(t^{2}+x^{2}\right)^{\frac{n+1}{2}}}, \quad t>0, x \in \mathbb{R}^{n},
$$

but its direct evaluation is not easy except for the case of low dimensions $n$.

We give here an easy proof based on the reciprocal of $(\mathrm{T})$ :

$$
e^{-z \sqrt{\tau}}=\int_{0}^{\infty} e^{-\tau t} \frac{z}{\sqrt{4 \pi t^{3}}} e^{-\frac{z^{2}}{4 t}} d t, \quad z>0, \operatorname{Re} \tau \geq 0 .
$$

Substituting $e^{-t \sqrt{\xi^{2}}}$ in $(\mathrm{P})$ by its integral expression, we obtain

$$
\begin{aligned}
P_{n}(t, x) & =\frac{1}{(2 \pi)^{n}} \int_{\mathbb{R}^{n}} e^{i x \xi} d \xi \int_{0}^{\infty} \frac{t}{\sqrt{4 \pi s^{3}}} e^{-\frac{t^{2}}{4 s}} e^{-s|\xi|^{2}} d s \\
& =\int_{0}^{\infty} \frac{t}{\sqrt{4 \pi s^{3}}} e^{-\frac{t^{2}}{4 s}} \frac{1}{(4 \pi s)^{\frac{n}{2}}} e^{-\frac{|x|^{2}}{4 s}} d s \\
& =\frac{t}{\left(t^{2}+x^{2}\right)^{\frac{n+1}{2}}} \frac{1}{\pi^{\frac{n+1}{2}}} \int_{0}^{\infty} u^{\frac{n+1}{2}-1} e^{-u} d u,
\end{aligned}
$$

where $u=\frac{t^{2}+|x|^{2}}{4 s}$ and the area $\omega_{n+1}$ of the unit sphere in $\mathbb{R}^{n+1}$ is $\frac{2 \pi^{(n+1) / 2}}{\Gamma((n+1) / 2)}$.

We also note that, similarly to 19 and 20, , we have

$$
P_{n}(t, x)=-2 \frac{\partial}{\partial t} N_{n+1}(t, x),
$$

where $N_{n+1}(t, x)$ is the Newton kernel, that is, the symmetric fundamental solution to the Laplacian:

$$
N_{n+1}(t, x)= \begin{cases}\frac{-1}{2 \pi} \log \sqrt{t^{2}+x^{2}}, & n=1, \\ \frac{1}{\omega_{n+1}(n-1)}\left|\sqrt{t^{2}+x^{2}}\right|^{-n+1}, & n>1 .\end{cases}
$$

6. The abstract Fatou theorem. If $\phi(x)$ is a function in $L^{p}\left(\mathbb{R}^{n}\right), 1 \leq p \leq \infty$, then the Poisson integral $u(t, x)=P(t) \phi(x)$ is a harmonic function in the upper half space $\left\{(t, x) \in \mathbb{R}^{n+1}: t>0\right\}$, whose $L^{p}$-norm is uniformly bounded, that is, it satisfies

$$
\left\{\begin{array}{l}
\frac{\partial^{2} u}{\partial t^{2}}=-\Delta u, \quad t>0, x \in \mathbb{R}^{n} \\
\sup _{t>0}\|u(t, \cdot)\|_{L^{p}\left(\mathbb{R}^{n}\right)}<\infty
\end{array}\right.
$$

Conversely, Fatou [6], for $p=\infty$, and Hardy-Littlewood [8], for $1 \leq p<\infty$, have shown that if $u(t, x)$ satisfies $(23)$, then it has the boundary value

$$
u(0, x)=\phi(x), \quad x \in \mathbb{R}^{n},
$$

and $u(t, x)=P(t) \phi(x)$. 
The calculation (22) shows that the Poisson semigroup $P(t) \phi(x)$ on $L^{p}\left(\mathbb{R}^{n}\right)$ for $1 \leq$ $p \leq \infty$, is related to the Gauss semigroup

$$
G(t) \phi(x)=\frac{1}{(4 \pi t)^{\frac{n}{2}}} \int_{\mathbb{R}^{n}} e^{-\frac{|x-y|^{2}}{4 t}} \phi(y) d y,
$$

by

$$
P(t) \phi(x)=\int_{0}^{\infty} \frac{t}{\sqrt{4 \pi s^{3}}} e^{-\frac{t^{2}}{4 s}} G(s) \phi(x) d s .
$$

Let, more generally, $G(t)=\exp (-t A)$ be any bounded strongly continuous semigroup on a Banach space $X$ with the generator $-A$. Then, the integral defines another bounded strongly continuous semigroup $P(t)$. Balakrishnan [1] defined the square root $\sqrt{A}$ of the operator $A$ as the negative of the generator of the semigroup $P(t)$, so that $P(t)=$ $\exp (-t \sqrt{A})$.

Later Balakrishnan [2] and Komatsu [13] have developed the theory of fractional powers $A^{\alpha}, \operatorname{Re} \alpha>0$, for non-negative operators $A$, that is, closed linear operators in a Banach space $X$ such that the negative real line $(-\infty, 0)$ is included in the resolvent set of $A$ and, moreover, the resolvent $(\lambda+A)^{-1}$ has the uniform estimate

$$
M_{0}=\sup _{0<\lambda<\infty}\left\|\lambda(\lambda+A)^{-1}\right\|<\infty .
$$

The negative $-A$ of a non-negative operator $A$ with dense domain in a Banach space $X$ does not necessarily generate a continuous semigroup but $-\sqrt{A}$ does a bounded holomorphic semigroup $\exp (-t \sqrt{A})$, so that for any $\phi \in X, u(t)=\exp (-t \sqrt{A}) \phi$ is a solution of

$$
\left\{\begin{array}{l}
\frac{d^{2}}{d t^{2}} u(t)=A u(t), \quad t \in(0, \infty), \\
\sup _{t>0}\|u(t, \cdot)\|_{X}<\infty
\end{array}\right.
$$

with the initial value $u(0)=\phi$.

Conversely, if $X$ is reflexive or if it is the dual $X_{*}^{\prime}$ of a Banach space $X_{*}$ and if $A$ is the dual of a non-negative operator $A_{*}$ in $X_{*}$ with dense domain, then we can find for any solution of 24) a unique $\phi \in X$ such that $u(t)=\exp (-t \sqrt{A}) \phi \quad(2]$ Theorem 6.1, [21]).

If we apply this theorem to the differential operator $A=C R \partial / \partial t$, we obtain the uniqueness of solutions to Thomson's problem (5) under the additional condition

$$
\sup _{x>0}\|v(\cdot, x)\|_{L^{p}}<\infty
$$

for a $1 \leq p \leq \infty$.

Acknowledgments. This work was supported in part by JSPS Grant-in-Aid for Scientific Research (C) 20540107.

\section{References}

[1] A. V. Balakrishnan, An operational calculus for infinitesimal generators of semigroups, Trans. Amer. Math. Soc. 91 (1959), 330-353. 
[2] A. V. Balakrishnan, Fractional powers of closed operators and the semigroups generated by them, Pacific J. Math. 10 (1960), 419-437.

[3] T. J. l'A. Bromwich, Normal coordinates in dynamical systems, Proc. London Math. Soc. 15 (1916), 401-448.

[4] J. R. Carson, The Heaviside operational calculus, Bull. Amer. Math. Soc. (N.S.) 32 (1926), $43-68$.

[5] P. A. M. Dirac, The physical interpretation of the quantum dynamics, Proc. R. Soc. Lond. Ser. A Math. Phys. Eng. Sci. 113 (1926-27), 621-641.

[6] P. Fatou, Séries trigonométriques et séries de Taylor, Acta Math. 30 (1906), 335-400.

[7] J. Fourier, Théorie Analytique de la Chaleur, Firmin Didot, Paris, 1822; Jacques Gabay, Sceaux, 1988.

[8] G. H. Hardy and J. E. Littlewood, Some properties of fractional integrals, II, Math. Z. 34 (1932), 403-439.

[9] O. Heaviside, Electrical Papers, Vol. II, The Electrician, London, 1892; Chelsea, New York, 1970.

[10] O. Heaviside, On operators in mathematical physics, Proc. R. Soc. Lond. Ser. A Math. Phys. Eng. Sci. 52 (1893), 504-529 and 54 (1894), 105-143.

[11] O. Heaviside, Electromagnetic Theory, Vol. II, The Electrician, London, 1899; Chelsea, New York, 1971.

[12] H. Jeffreys and B. Jeffreys, Methods of Mathematical Physics, 3rd ed., Cambridge Univ. Press, 1956.

[13] H. Komatsu, Fractional powers of operators, Pacific J. Math. 19 (1966), 285-346.

[14] H. Komatsu, Ultradistributions, I, Structure theorems and a characterization, J. Fac. Sci., Univ. Tokyo, Sec IA, 20 (1973), 25-105.

[15] H. Komatsu, Laplace transforms of hyperfunctions - A new foundation of the Heaviside calculus, J. Fac. Sci. Univ. Tokyo, Sec. IA 34 (1987), 805-820.

[16] H. Komatsu, Operational calculus, hyperfunctions and ultradistributions, in: Algebraic Analysis, Vol. 1, Academic Press, San Diego, 1988, 357-372.

[17] H. Komatsu, Operational calculus and Japan, in: Generalized Functions and Convergence, World Scientific, Singapore, 1990, 59-70.

[18] H. Komatsu, Operational calculus and semi-groups of operators, in: Functional Analysis and Related Topics, 1991 (Kyoto), Lecture Notes in Math. 1540 (1993), 213-234.

[19] H. Komatsu, Multipliers for Laplace hyperfunctions - A justification of Heaviside's rules, Trudy Mat. Inst. Steklov 203 (1994), 323-333; Proc. Steklov Inst. Math. 3 (1995), 271-279.

[20] H. Komatsu, Solution of differential equations by means of Laplace hyperfunctions, in: Structure of Solutions of Differential Equations, World Scientific, Singapore, 1996, 227252 .

[21] H. Komatsu, The abstract Fatou theorem and the theory of signal transmission on Thomson cables, to appear in Hokkaido Math. J.

[22] P. Lévy, Le calcul symbolique d'Heaviside, Bull. Sci. Math. 50 (1926), 174-192.

[23] J. Mikusiński, Operational Calculus, 2nd ed., Pergamon Press, 1983.

[24] B. van der Pol and H. Bremmer, Operational Calculus Based on the Two-sided Laplace Integral, 2nd ed., Cambridge Univ. Press, 1955.

[25] L. Schwartz, Théorie des Distributions, Hermann, Paris, 1950-51.

[26] L. Schwartz, Transformation de Laplace des distributions, Medd. Lunds Math. Sem., Suppl. (1952), 196-206. 
[27] J. Sebastião e Silva, Les fonctions analytiques comme ultradistributions dans le calcul opérationnel, Math. Ann. 136 (1958), 58-96.

[28] W. Thomson, On the theory of the electric telegraph, Proc. Royal Soc. London, 1855; Mathematical and Physical Papers, vol. 2, 61-78.

[29] E. T. Whittaker, Oliver Heaviside, Bull. Calcutta Math. Soc. 20 (1928), 199-220; reprint in: O. Heaviside: Electromagnetic Theory, Vol. I, 3rd ed., Chelsea, New York, 1971. 
